\title{
What happens if a drunkard is asked to walk a full circle around a lake in a short time?
}

\author{
Brownian flights over a circle \\ Authors: Alexander Vladimirov, Senya Shlosman, Sergei Nechaev \\ Phys. Rev. E 102, 012124 (2020) \\ Recommended with a Commentary by Alexander Y. Grosberg, \\ Department of Physics and Center for Soft Matter Research, \\ New York University, 726 Broadway, New York, NY 10011
}

A random walker during time $t$ moves distance that scales as $t^{1 / 2}$. This critical exponent of $\nu=1 / 2$ shows up in innumerable variety of circumstances in physics, sometimes in disguise, such as, e.g., the decay of probability distribution for the classical gambler's ruin problem (first passage time distribution) as $\sim t^{-3 / 2}$ is in fact $\sim t^{-(2+d) \nu}$.

Kardar-Parisi-Zhang model of a growing surface gives the most widely known example of fundamentally different and independent scaling $\sim t^{2 / 3}$ (see, e.g. textbook [1, section 9.6]).

In the recent series of papers $[2,3,4,5]$, S.Nechaev and co-authors suggested an unexpected and possibly deep mathematical connection between these two realms. Specifically, they considered the problem of a random walk on the plane outside of an excluded round disk conditioned on returning back to the starting point after specified time $t$ and making exactly one (or any specified larger number) of turns around the obstacle. This is illustrated in the Figure. A similar problem was considered also independently by Meerson and Smith [6], with a similar results. In the limit when obstacle radius $R$ is much larger than $R_{0}$, root mean square of the distance that random walker would have traveled in the given time $t$ in a free plane without obstacles, the trajectory of the random walk is strongly stretched by the condition to wind around the obstacle. It was found in the papers $[2,3,4,5,6]$ that the walk trajectory is in this case localized in a

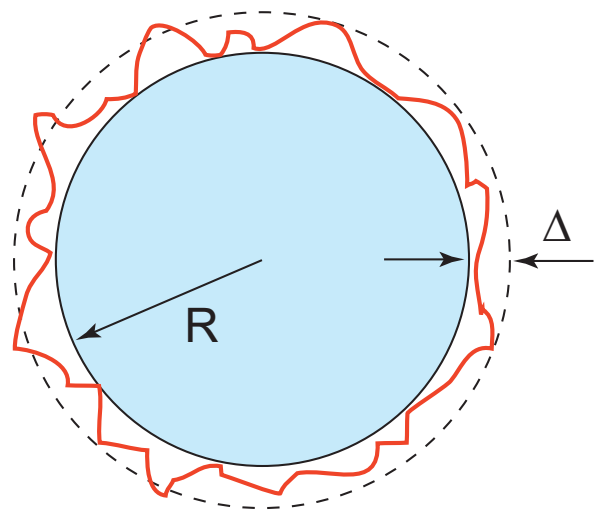

Figure 1: Random walk (in red) encircling an obstacle of a large radius $R$ with a fixed winding number (in this illustration 1). The random walker deviates from the surface of the obstacle by a small distance $\Delta$. narrow strip of the width

$$
\Delta \sim R_{0}^{4 / 3} / R^{1 / 3}
$$

in other words, $\Delta \sim t^{2 / 3}-$ KPZ-like scaling. Note that $\Delta \ll R_{0} \ll R$. 
The works $[2,3,4,5]$ (unlike [6]) are formulated mostly in terms of a ring polymer stretched around a disk in the limit when disk circumference is large for the given polymer length. Present writer found this terminology quite confusing, because polymer chains, unlike random walks, have inherently limited extensibility, and at strong stretching their similarity with random walks breaks down (see, e.g., [7]). For this reason, when polymer length is compared to $2 \pi R$, my temptation is to think of a polymer with a large, but finite number of segments $N$. In fact, I think that the result (1) is completely unrelated to this issue of finite extensibility, which can be understood by the following simple argument.

In polymer language, the width $\Delta$ over which the chain is delocalized must result from the minimization of the following free energy:

$$
\frac{F}{k_{B} T} \sim \frac{N b^{2}}{\Delta^{2}}+\frac{(2 \pi(R+\Delta))^{2}}{N b^{2}} .
$$

Here the first term describes entropy penalty for confining chain within the strip of the width $\Delta$, while the second term arises from entropy penalty for stretching the chain. The only slightly non-trivial fact here is that chain is stretched not just to the circumference length $2 \pi R$, but to a slightly longer length about $2 \pi(R+\Delta)$. Note that both terms are written for a purely Gaussian chain, $b$ being the monomer size, and no finite extensibility corrections are taken into account. Minimization of this free energy (in case $\Delta \ll R$ ) yields the result $\Delta^{3} \sim \frac{N^{2} b^{4}}{R}$ which is identical to (1), modulo notations.

Of course, for the benefit of people thinking in terms of random walks and not polymers, the above argument is easily reformulated: the statistical weight to be maximized is $\exp \left[-\frac{D t}{\Delta^{2}}-\frac{(2 \pi(R+\Delta))^{2}}{D t}\right]$, where $D$ is the diffusion coefficient of the random walker, and $t$ is the time until trajectory closure.

Given that the result (1) arises from the regular random walks considerations, and, therefore, its scaling appears to be yet another disguise* of the usual random walk exponent $\nu=1 / 2$, the mathematical links of this scaling to the realm of KPZ become even more tantalizing.

\section{References}

[1] Mehran Kardar "Statistical Physics of Fields", Cambridge University Press, 2007.

[2] Sergei Nechaev, Kirill Polovnikov, Senya Shlosman, Alexander Valov, Alexander Vladimirov "Anomalous 1D fluctuations of a simple 2D random walk in a large deviation regime" arXiv:1801.03912; Phys. Rev. E 99, 012110 (2019).

[3] Alexander Vladimirov, Senya Shlosman, Sergei Nechaev "Brownian flights over a circle" arXiv:2002.09965; Phys. Rev. E 102, 012124 (2020).

[4] Alexander Gorsky, Sergei Nechaev, Alexander Valov "Equilibrium mean-field-like statistical models with KPZ scaling" arXiv:2005.02382 (2020).

\footnotetext{
*In fact, $\frac{2}{3}=\frac{2 \nu+1}{\nu+1} \nu$.
} 
[5] Sergei Nechaev, Alexander Valov "Fixman problem revisited: When fluctuations of inflated ideal polymer loop are non-Gaussian?" arXiv:2011.07802 (2020).

[6] Baruch Meerson, Naftali R. Smith "Geometrical optics of constrained Brownian motion: three short stories", J. Phys. A: Math. Theor. 52, 415001 (2019).

[7] S.B. Smith, L. Finzi, and C. Bustamante "Direct mechanical measurements of the elasticity of single DNA molecules by using magnetic beads", Science, 258, 1122-1126 (1992). 